\title{
Expression profiles of microRNAs and their target genes in papillary thyroid carcinoma
}

\author{
XIN LIU ${ }^{1}$, MENGZI HE ${ }^{2}$, YUFEI HOU ${ }^{3}$, BING LIANG ${ }^{2}$, LONGYU ZHAO ${ }^{1}$, \\ SHUMEI MA $^{2}$, YAQIN YU ${ }^{4}$ and XIAODONG LIU ${ }^{2,5}$ \\ ${ }^{1}$ Department of Bioinformatics and Statistics, School of Public Health, Jilin University; ${ }^{2}$ Key Laboratory of Radiobiology \\ (Ministry of Health), School of Public Health, Jilin University, Changchun 130021; ${ }^{3}$ School of Public Health, Jilin University, \\ Changchun 130021; ${ }^{4}$ Department of Epidemiology, School of Public Health, Jilin University, Changchun 130021; \\ ${ }^{5}$ Department of Radiology and Radiation Oncology, China-Japan Union Hospital, Changchun 130021, P.R. China
}

Received October 19, 2012; Accepted December 10, 2012

DOI: 10.3892/or.2013.2263

\begin{abstract}
The incidence of thyroid cancer has recently experienced a rapid increase in China, and papillary thyroid carcinoma (PTC) accounts for nearly $80 \%$ of human thyroid cancers. In the present study, the differential expression of microRNAs (miRNAs) and their target genes were identified in order to analyze the potential roles of miRNAs as biomarkers and in papillary thyroid carcinogenesis. One hundred and twenty-six PTC samples were collected from patients at the China-Japan Union Hospital, China, and the gene/miRNA expression profiles were examined with Illumina BeadChips and verified by real-time RT-PCR. Gene Ontology (GO) categories were determined, and pathway analysis was carried out using KEGG. miRNA target genes were predicted by implementing three computational analysis programs: TargetScanS, DIANA-microT and PicTar. Two hundred and forty-eight miRNAs and 3,631 genes were found to be significantly deregulated (gene, $\mathrm{P}<0.05$; miRNA, $\mathrm{P}<0.01$ ) in PTC tissues when compared with their matching normal thyroid tissues. hsa-miR-206 (target gene, MET), hsa-miR-299-3p (target gene, ITGAV), hsa-miR-101 (target gene, ITGA3), hsa-miR103 (target gene, ITGA2), hsa-miR-222 (target genes, KIT and AXIN2), hsa-miR-15a (target genes, AXIN2 and FOXO1) and hsa-miR-221 (target gene, KIT) were identified. Together
\end{abstract}

Correspondence to: Dr Xiaodong Liu or Dr Shumei Ma, Key Laboratory of Radiobiology (Ministry of Health), School of Public Health, Jilin University, 1163 Xinmin Street, Changchun 130021, P.R. China

E-mail: liuxiaod@jlu.edu.cn

E-mail:shmm2001@yahoo.com.cn

Dr Yaqin Yu, Department of Epidemiology, School of Public Health, Jilin University, Changchun 130021, P.R. China

E-mail: yuyq@jlu.edu.cn

Key words: papillary thyroid carcinoma, miRNA, biomarker, gene profile with the functions of the target genes, we further elucidated the role of miRNAs in papillary thyroid carcinogenesis and suggest the use of miRNAs as biomarkers for early diagnosis. Our findings provide the basis for future studies in the field of miRNA-based cancer therapy.

\section{Introduction}

Thyroid cancer is the most common endocrine malignancy and recently its worldwide incidence is rapidly rising $(1,2)$. It is considered as a highly prevalent malignant disease. The American National Cancer Institute reported that during the years of 2005-2009, the median age at diagnosis for thyroid cancer was 50 years, and 56,460 new cases and 1,780 deaths from thyroid cancer were estimated in the United States in 2012. There are four main types of thyroid cancer: papillary, follicular, medullary and anaplastic thyroid cancer. Papillary thyroid carcinoma (PTC) accounts for approximately $80 \%$ of human thyroid cancers. Thyroid cancer constitutes one of the most curable tumors; however, the differential diagnosis can often be elusive. Although fine-needle aspiration biopsy is currently the most useful diagnostic technique for evaluating a thyroid nodule, preoperative diagnosis of thyroid nodules is frequently imprecise, with up to $30 \%$ of fine-needle aspiration biopsy cytology samples reported as 'suspicious' or 'indeterminate' (3). Therefore, molecular-based diagnostic approaches are needed for the preoperative distinction of these lesions.

It has been demonstrated that numerous miRNAs (miRNAs) are transcriptionally upregulated in PTC compared with unaffected thyroid tissue. miRNAs are small ( 21 nucleotides long) non-coding RNAs, which regulate gene expression at the posttranscriptional level and play important roles in a multitude of biological processes, including tumorgenesis (4,5). A specific miRNA may function either as an oncogene or as a tumor suppressor by regulating the expression of target oncogene(s) and tumor suppressors, respectively. Generally, miRNAs bind to the 3'-untranslated regions (3' UTRs) of target mRNAs, leading to mRNA degradation or repression of translation. In the present study, miRNA and gene profiles were analyzed to identify the differential expression of miRNAs and target 
genes in PTC vs. normal thyroid tissues in an attempt to elucidate the potential roles of miRNAs as biomarkers and in the carcinogenesis of PTC.

\section{Materials and methods}

Tumor specimens. A total of 126 thyroid samples were collected from PTC patients undergoing thyroidectomy at China-Japan Union Hospital, China between 2010 and 2011. After surgical excision, the specimens including PTC and their matching normal thyroid tissues were snap frozen in liquid nitrogen until use. The pathological types of the tumors were verified by pathologists. All tissues were obtained following prior informed consent from each patient.

RNA extraction. Total RNA was isolated using TRIzol reagent (Invitrogen, Carlsbad, CA, USA). The quantity and the integrity of extracted RNA were determined by UV spectrophotometer. Total RNA was quantified by formaldehyde denaturing gel electrophoresis.

Real-time reverse transcription-PCR. Real-time reverse transcription-PCR was used to verify the gene expression using Stratagene Mx3000P (Japan). Reverse transcription was performed with $500 \mathrm{ng}$ RNA for the $10-\mu 1$ reverse transcription reaction, and $1 \mu \mathrm{l} \mathrm{cDNA}$ was then used for each $25-\mu \mathrm{l}$ reaction mixture that contained an optimal concentration of primers and SYBR-Green Supermix (SYBR Premix Ex Taq II; Takara Co., Osaka, Japan). A total of 45 cycles was performed, consisting of $20 \mathrm{sec}$ at $95^{\circ} \mathrm{C}$ and $20 \mathrm{sec}$ at $60^{\circ} \mathrm{C}$ for each cycle. GAPDH was used as an internal control.

Illumina BeadChip and data analysis. Total RNAs were hybridized to the Illumina Human Genome BeadChip Array and miRNA BeadChip (Illumina). The arrays were scanned on an Illumina BeadStation 500 System, and the hybridization data were analyzed using Illumina BeadStudio software. The following filtering criteria were used for selection of differentially expressed genes: for a positive gene or miRNA in the tumor $(\mathrm{T})$ or control $(\mathrm{N})$ (matching normal thyroid tissues): detection, $\mathrm{P}<0.05$; ratio (groupT/groupN) $>2$ or ratio (groupT/ groupN) $<0.5$. All data were MIAME compliant, and the raw data were deposited in the ArrayExpress database along with the normalized data.

Gene Ontology $(G O)$ category and pathway analysis. The differentially expressed genes were analyzed for inclusion in GO categories and pathways. Categorization in significant biological processes was performed using tools of the Gene Ontology project (http://www.geneontology.org). The test of statistical significance considers the number of differentially expressed genes found in each category compared with the total number of genes in the category represented on the chip. The pathway analysis was carried out using the Kyoto Encyclopedia of Genes and Genomes (KEGG) database. Two-sided Fisher's exact test and the $\chi^{2}$ test were used to classify the GO category and pathway, and the false discovery rate (FDR) was calculated to correct the P-value. P-value $<0.05$ and FDR $<0.05$ were used as a threshold to select significant GO categories and KEGG pathways.
Table I. Result of the gene and miRNA chips.

\begin{tabular}{lcc}
\hline & Genes & miRNAs \\
\hline Upregulated & 2,013 & 180 \\
Downregulated & 1,618 & 68 \\
Total & 3,631 & 248 \\
\hline
\end{tabular}

The gene network analysis of the differentially expressed genes involved in significant pathways was carried out using the KEGG database. Interactions of genes in the database were analyzed and gene networks were established.

Prediction of miRNA target genes. In the present study, three websites including TargetScanS (http://www.targetscan.org/), DIANA-microT (http://diana.cslab.ece.ntua.gr/microT/) and PicTar (http://pictar.mdc-berlin.de/) were used to predict miRNA targets. A gene that was predicted in two or three of these target prediction programs was selected as a potent candidate.

\section{Results}

miRNA and gene expression in PTC tissues. Two hundred and forty-eight miRNAs and 3,631 genes were significantly differentially expressed (for genes, $\mathrm{P}<0.05$; miRNA, $\mathrm{P}<0.01$ ) between PTC tissues and the matching normal thyroid tissues. Of these 248 miRNAs, 180 were overexpressed and 68 were underexpressed in PTC tissues. Of the 3,631 genes 2,013 were overexpressed and 1,618 were underexpressed in PTC tissues (Table I). To verify the results of the chips, real-time RT-PCR was carried out for verification.

Deregulated genes involved in carcinogenesis. After the differentially expressed genes were analyzed for inclusion in GO categories and KEGG pathways, 68 of the deregulated genes were found to be involved in tumor signaling pathways or to play important roles in cancer (data not shown) including the Wnt signaling pathway, mTOR signaling pathway, JAK-STAT signaling pathway, ErbB signaling pathway, MAPK signaling pathway, VEGF signaling pathway, PPAR signaling pathway, adherens junction, ECM-receptor interaction, focal adhesion, cytokine-cytokine receptor interaction, apoptosis and the cell cycle (Fig. 1).

Matching target genes were determined for the deregulated miRNAs in PTC. The most popular three target prediction programs were used to identify putative target genes for the 248 deregulated miRNAs in PTC. Matching target genes for 88 differentially expressed miRNAs in PTC were successfully found (data not shown). Of the 88 miRNAs, 64 were upregulated and 24 were downregulated. Using the results of the target genes, we compared the data with the deregulated genes from the gene BeadChip data to find those genes similarly expressed. After the data analysis, 578 target genes for the differentially expressed miRNAs in PTC were finally identified. Of these 578 genes, 298 were upregulated and 280 were downregulated. 


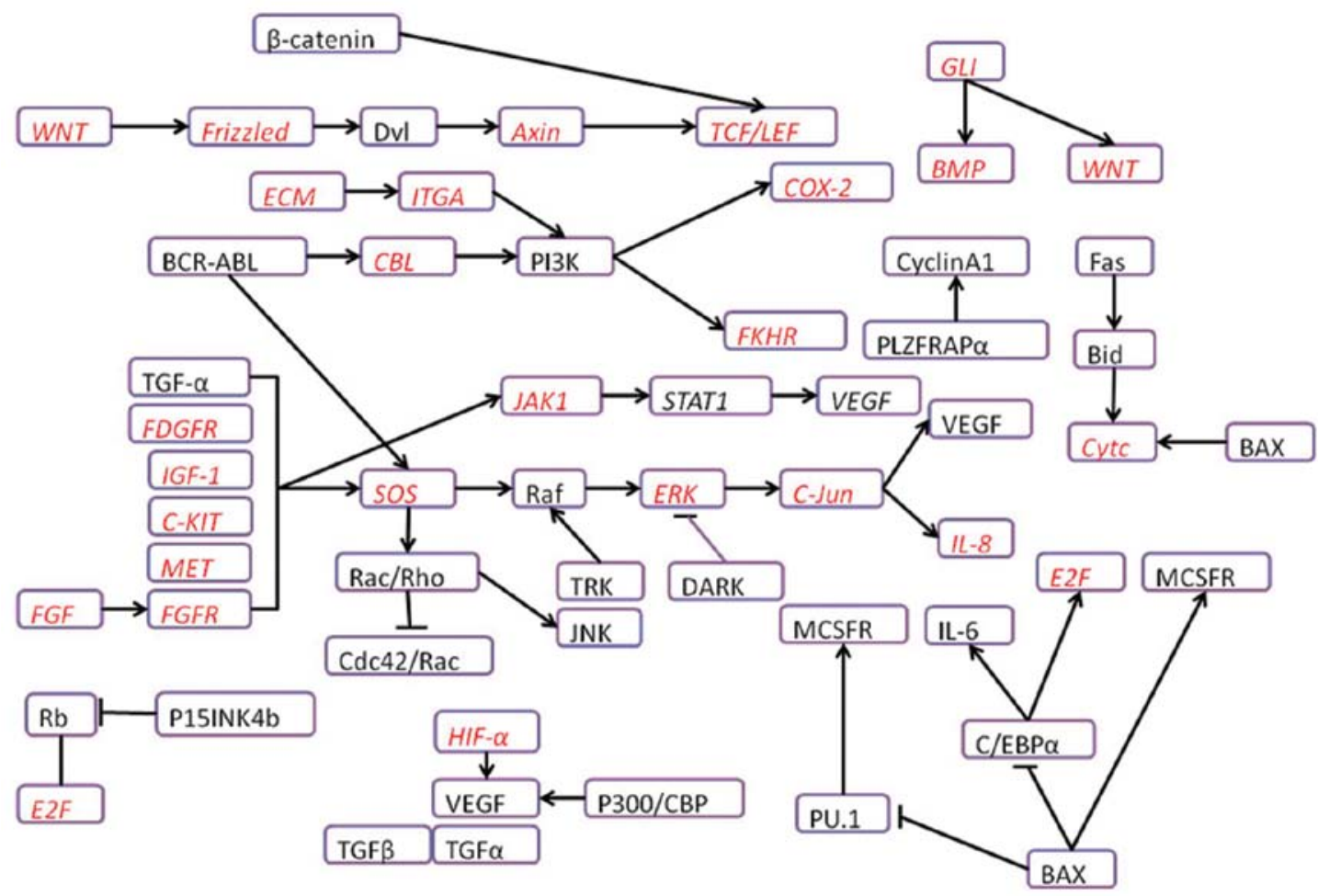

Figure 1. Gene network of the differentially expressed genes in papillary thyroid carcinoma (PTC) involved in cancer-related pathways. We identified 68 deregulated genes in PTC tissues known to be involved in tumor signaling pathways or to play important roles in cancer according to the KEGG database. The target genes for the deregulated miRNAs in PTC are indicated in italics.

Table II. Downregulated target genes of miRNAs involved in cancer-related pathways.

Target genes (downregulated)

miRNAs (upregulated)

\section{FZD8}

WNT11

RUNX1T1

PDGFRA

KIT

JUN

IGF1

GLI3

FZD8

FOXO1

FGF11

AXIN2
hsa-miR-99b

hsa-miR-340

hsa-miR-340

hsa-miR-454, hsa-miR-340, hsa-miR-196a, hsa-miR-181b

hsa-miR-222, hsa-miR-221

hsa-miR-524-5p

hsa-miR-98, hsa-miR-454, hsa-miR-340, hsa-miR-18a, hsa-let-7g, hsa-let-7e

hsa-miR-506

hsa-miR-506

hsa-miR-578, hsa-miR-524-5p, hsa-miR-381, hsa-miR-330-3p, hsa-miR-15a hsa-miR-98, hsa-let-7g, hsa-let-7e

hsa-miR-222, hsa-miR-15a
Association between the deregulated miRNAs and their putative target genes. To find a correlation between miRNAs and their target genes in cancer pathways of PTC, the 578 target genes of the deregulated miRNAs were compared with 68 genes which participate in cancer pathways. Twenty-eight genes and the related signaling pathways were consistent. We found that the target genes of the deregulated miRNAs were involved in the Wnt signaling pathway, mTOR signaling pathway, JAK-STAT signaling pathway, ErbB signaling pathway, MAPK signaling pathway, VEGF signaling pathway,
ECM-receptor interaction, focal adhesion, cytokine-cytokine receptor interaction, apoptosis and the cell cycle (Fig. 1). Of these 28 genes, 16 were upregulated and 12 were downregulated. We further investigated the relationship between the miRNAs and their target genes in the cancer pathways of PTC. There were 29 miRNAs and 28 genes. Of these 29 miRNAs, 17 were upregulated (12 weakly expressed related target genes) and 12 were downregulated (16 highly expressed related target genes) (Tables II and III). We next sought to identify mRNAs and their putative target genes which were 
Table III. Upregulated target genes of miRNAs involved in cancer-related pathways.

\begin{tabular}{ll}
$\begin{array}{l}\text { Target genes } \\
\text { (upregulated) }\end{array}$ & \multicolumn{1}{c}{\begin{tabular}{c}
\multicolumn{1}{c}{$\begin{array}{c}\text { miRNAs } \\
\text { (downregulated) }\end{array}$} \\
FGFR3
\end{tabular}} \\
WNT5A & hsa-miR-99a \\
hOS1 & hsa-miR-374a \\
PTGS2 & hsa-miR-101 \\
MET & hsa-miR-206 \\
MAPK1 & hsa-miR-206, hsa-miR-140-3p, hsa-miR-101 \\
LEF1 & hsa-miR-372 \\
JAK1 & hsa-miR-30e \\
ITGAV & hsa-miR-363, hsa-miR-299-3p, hsa-miR-135b \\
ITGA3 & hsa-miR-101 \\
ITGA2 & hsa-miR-107, hsa-miR-103 \\
IL8 & hsa-miR-372 \\
HIF1A & hsa-miR-372 \\
FN1 & hsa-miR-206 \\
E2F2 & hsa-miR-372 \\
CBL & hsa-miR-206 \\
\hline
\end{tabular}

significantly differentially expressed and which had both a high ratio (upregulated ratio $>2$ or downregulated ratio $<0.5$ ) and a high signal (either signal value $>200$ ). Finally, hsa-miR206 (target gene, MET) hsa-miR-299-3p (target gene, ITGAV), hsa-miR-101 (target gene, ITGA3) hsa-miR-103 (target gene, ITGA2), hsa-miR-222 (target genes, KIT and AXIN2), hsamiR-15a (target genes, AXIN2 and FOXO1) and hsa-miR-221 (target gene, KIT) were identified (Table IV).

To verify the results, hsa-miR-222, hsa-miR-15a and their consistent putative target gene AXIN2 were validated by real-time PCR. hsa-miR-222 was $94.11 \%$ upregulated, hsa-miR-15a was $64.7 \%$ upregulated and AXIN2 was $80 \%$ downregulated between PTC and their matching normal thyroid tissues. We then compared the results between miRNA and putative target gene, and we found that $94.12 \%$ of the samples had a regulatory relationship between hsa-miR-222 and AXIN2, and 70.59\% had a regulatory relationship between hsa-miR-15a and AXIN2 (Table V).

\section{Discussion}

miRNAs play important roles in multiple biological and metabolic processes, such as cell differentiation, proliferation, survival and malignancies (6-13). They are endogenous single-stranded non-coding RNAs 22 nucleotides in length which suppress gene expression by selectively binding to the complementary 3' UTR of their target mRNAs through imperfect base-pairing.

In order to improve the accuracy of the predicted target genes, we compared the gene expression profile with the miRNA profile and limited the target genes to those known to be involved in cancer. The miRNAs and their target genes based on the difference ratio values and expression intensity were both at high levels. According to the functions of the target genes, we further speculated the role of the miRNAs in the specific PTC pathways.

In the present study, PTC samples were harvested and the gene expression and miRNA profiles were detected. Altogether 3,631 genes and 248 miRNAs were deregulated as noted from the chip data. We implemented KEGG software analysis and found that 68 of the deregulated genes play important roles in cancer or cancer-related signaling pathways. The target genes of the deregulated miRNAs in PTC tissue were then identified by network and compared with the differentially expressed genes in the gene chip. In order to further narrow the scope of the study, we ascertained whether the 68 genes we identified from the differentially expressed genes by KEGG, were the target genes we had predicted. Finally, hsa-miR-206 (target gene, MET), hsa-miR-299-3p (target gene, ITGAV), hsa-miR-101 (target gene, ITGA3), hsa-miR-103 (target gene, ITGA2), hsa-miR-222 (target genes, KIT and AXIN2) hsa-miR-15a (target genes, AXIN2 and FOXO1) and hsa-miR-221 (target gene, KIT) were identified as either miRNAs or genes with a high ratio and high signal in the chips.

In our study, hsa-miR-222, hsa-miR-15a and their consistent putative target gene AXIN2 were verified by real-time PCR. hsa-miR-222 was $94.11 \%$ upregulated, consistent with previous research, hsa-miR-15a was $64.7 \%$ upregulated

Table IV. Target genes of the differentially expressed miRNAs in PTC.

\begin{tabular}{llccccc}
\hline & & \multicolumn{3}{c}{ Target prediction programs } & & \\
\cline { 3 - 4 } miRNA & Target gene & PicTar & TargetScanS & DIANA-microT & 3' UTR & miRNA upregulated/downregulated \\
\hline hsa-miR-221 & KIT & & $\sqrt{ }$ & $\sqrt{ }$ & UACAUCG & Upregulated \\
hsa-miR-222 & KIT & & $\sqrt{ }$ & $\sqrt{ }$ & UACAUCG & Upregulated \\
hsa-miR-222 & AXIN2 & & $\sqrt{ }$ & $\sqrt{ }$ & UACAUCG & Upregulated \\
hsa-miR-15a & AXIN2 & $\sqrt{ }$ & $\sqrt{ }$ & & ACGACGA & Upregulated \\
hsa-miR-15a & FOXO1 & & $\sqrt{ }$ & $\sqrt{ }$ & ACGACGA & Upregulated \\
hsa-miR-206 & MET & $\sqrt{ }$ & $\sqrt{ }$ & & UGUAAGG & Downregulated \\
hsa-miR-299-3p & ITGAV & $\sqrt{ }$ & $\sqrt{ }$ & & GGGUGUA & Downregulated \\
hsa-miR-101 & ITGA3 & & $\sqrt{ }$ & $\sqrt{ }$ & CAUGACA & Downregulated \\
hsa-miR-103 & ITGA2 & $\sqrt{ }$ & $\sqrt{ }$ & $\sqrt{ }$ & UACGACG & Downregulated \\
\hline
\end{tabular}


Table V. Results of the real-time PCR.

\begin{tabular}{lcccc}
\hline miRNA & Upregulated & Target gene & Downregulated & miRNA upregulated and target gene downregulated \\
\hline hsa-miR-222 & $94.11 \%$ & AXIN2 & $80 \%$ & $94.12 \%$ \\
hsa-miR-15a & $64.7 \%$ & AXIN2 & $80 \%$ & $70.59 \%$ \\
\hline
\end{tabular}

and AXIN2 was $80 \%$ downregulated, and we found that $94.12 \%$ of the samples had regulatory relationships between hsa-miR-222 and AXIN2, and 70.59\% between hsa-miR-15a and AXIN2. The consistent trend suggested that the microarray assays were reliable. It was previously reported that hsa-miR-221 and hsa-miR-222 were upregulated in PTC tissues (14-18), consistent with our results, while hsa-miR-15a and hsa-miR-299 were downregulated (19). At the mRNA levels, MET was demonstrated to be upregulated (20) and KIT was downregulated $(21,22)$. In thyroid oncocytic tumors, ITGAV was found to be upregulated (23). The putative target gene of hsa-miR-221 and hsa-miR-222 has been identified as KIT, a tyrosine kinase receptor that plays an important role in cell growth and differentiation, acting as an oncogene in many types of cancers $(24,25)$. The above-mentioned results are consistent with our studies. Several deregulated miRNAs and genes have been identified by other researchers, such as hsa-miR-221, hsa-miR-222, hsa-miR-15a, hsa-miR-299, MET and KIT using the same gene array and real-time PCR. However, other miRNAs, such as hsa-miR-206, hsa-miR-101, hsa-miR-103, and the putative target genes ITGAV, ITGA2, ITGA3, FOXO1 and AXIN2 analyzed in our study were not reported previously. Their functions and significance still need further examination.

According to the different mechanisms of target genes in cancer development, ectopic manipulation of miRNA expression has been recently suggested as a novel therapeutic modality. In our study, the target genes are known to widely participate in the cancer process.

Met, the receptor for hepatocyte growth factor (HGF), is an oncogene that encodes the c-Met protein. c-Met protein is a hepatocellular growth factor receptor and is known to be responsible for the motility and mitogenesis of epithelial cells, including cancer cells $(26,27)$. Met is frequently activated in these carcinomas and may favor tumor growth, and the abundance of Met expression may differentially regulate cell growth, morphogenesis and migration in response to HGF.

ITGA2, ITGA3 and ITGAV, members of the integrins, are invasion-related genes $(28,29)$. Integrins are transmembrane adhesion molecules that mediate cell-cell and cell-extracellular matrix attachment. Integrins regulate cell growth, proliferation, migration and apoptosis and as a consequence, have a potential role in tumor progression and metastasis (30). It has been demonstrated that upregulation of the integrin subclasses could in fact enhance neoplastic cell motility and vascular endothelial growth factor expression $(31,32)$. ITGAV encodes the integrin $\alpha$-chain $\mathrm{V}$ protein involved in cell adhesion (cell to cell junctions) and signal transduction. Overexpression of ITGAV was significantly linked to the invasion and metastasis of laryngeal and hypopharyngeal squamous cell carcinoma (33).
FOXO1, acting as a regulator of cell responses to oxidative stress, is required for cell transition from proliferative growth to quiescence (by similarity). It triggers the Akt-dependent phosphorylation and inactivation of FKHRL1 functioning, suppresses the transcription of death genes, such as the Fas ligand gene, and thereby promotes cell survival (34).

Axis inhibition protein 2 (AXIN2) is a negative regulator of the Wnt/ $\beta$-catenin pathway and functions by participating in a negative feedback loop to limit the duration and intensity of a Wnt-initiated signal. This effect was demonstrated in several solid tumors such as colorectal carcinoma, liver tumors, craniopharyngiomas, hepatoblastoma and rare pancreatic tumors (35). Although it has been accepted that altered Wnt signaling is a late event in thyroid cell transformation that affects anaplastic thyroid tumors, recent data suggest that it is also altered in PTC with RET/PTC mutations (36).

The genes described in the present report are associated with the carcinogenesis, unlimited growth and invasiveness. The low or high expression of specific genes leads to malignant transformation of normal thyroid cells, and tumor metastasis may consequently occur in the disease process. Regulation of gene expression by miRNAs may become an important artifice for the treatment of cancer. These genes can also serve as diagnostic biomarkers.

In summary, several thyroid cancer-related miRNAs and their target genes were identified. These include hsa-miR206 (target gene, MET), hsa-miR-299-3p (target gene, ITGAV), hsa-miR-101 (target gene, ITGA3), hsa-miR-103 (target gene, ITGA2), hsa-miR-222 (target genes, KIT and AXIN2), hsa-miR-15a (target genes, AXIN2 and FOXO1) and hsa-miR-221 (target gene, KIT). According to the functions of the target genes, we can further elucidate the role of miRNAs in papillary thyroid carcinogenesis and determine the potential use of miRNAs as biomarkers for early diagnosis. Due to the limited number of samples in our study, further research is recommended to further validate the accuracy and specificity of our data. Moreover, our findings provide the basis for future research in the field of miRNA-based cancer therapy.

\section{Acknowledgements}

We thank Dr Qased Abu Baker (The Second Hospital Affiliated to Jilin University, Changchun, China) for the help in editing this manuscript. This study was supported by an NSFC grant (30770649, 30970682), Research Fund for the Doctoral Program of Higher Education of China (20100061110070), Program for New Century Excellent Talents in the University, Jilin Provincial Science and Technology Development Project (201205008), Science and Technology Project of the Department of Health of Jilin Province (2010Z005), and Jilin 
University Doctoral Interdisciplinary Scientific Research Project (450060483092).

\section{References}

1. Leenhardt L, Grosclaude P and Cherie-Challine L: Increased incidence of thyroid carcinoma in France: a true epidemic or thyroid nodule management effects? Report from the French Thyroid Cancer Committee. Thyroid 14: 1056-1060, 2004.

2. Davies L and Welch HG: Increasing incidence of thyroid cancer in the United States, 1973-2002. JAMA 295: 2164-2167, 2006.

3. Prasad NB, Somervell H, Tufano RP, et al: Identification of genes differentially expressed in benign versus malignant thyroid tumors. Clin Cancer Res 14: 3327-3337, 2008.

4. Chen AY, Jemal A and Ward EM: Increasing incidence of differentiated thyroid cancer in the United States, 1988-2005. Cancer 115: 3801-3807, 2009.

5. Vriens MR, Suh I, Moses W and Kebebew E: Clinical features and genetic predisposition to hereditary nonmedullary thyroid cancer. Thyroid 19: 1343-1349, 2009.

6. Calin GA and Croce CM: MicroRNA-cancer connection: the beginning of a new tale. Cancer Res 66: 7390-7394, 2006.

7. Michael MZ, O'Connor SM, van Holst Pellekaan NG, Young GP and James RJ: Reduced accumulation of specific microRNAs in colorectal neoplasia. Mol Cancer Res 1: 882-891, 2003.

8. Calin GA, Ferracin M, Cimmino A, et al: A MicroRNA signature associated with prognosis and progression in chronic lymphocytic leukemia. N Engl J Med 353: 1793-1801, 2005.

9. Eis PS, Tam W, Sun L, et al: Accumulation of miR-155 and BIC RNA in human B cell lymphomas. Proc Natl Acad Sci USA 102 3627-3632, 2005.

10. Takamizawa J, Konishi H, Yanagisawa K, et al: Reduced expression of the let-7 microRNAs in human lung cancers in association with shortened postoperative survival. Cancer Res 64: 3753-3756, 2004.

11. Iorio MV, Ferracin M, Liu CG, et al: MicroRNA gene expression deregulation in human breast cancer. Cancer Res 65: 7065-7070, 2005.

12. Ciafrè SA, Galardi S, Mangiola A, et al: Extensive modulation of a set of microRNAs in primary glioblastoma. Biochem Biophys Res Commun 334: 1351-1358, 2005.

13. Chan JA, Krichevsky AM and Kosik KS: MicroRNA-21 is an antiapoptotic factor in human glioblastoma cells. Cancer Res 65: 6029-6033, 2005.

14. He H, Jazdzewski K, Li W, et al: The role of microRNA genes in papillary thyroid carcinoma. Proc Natl Acad Sci USA 102: 19075-19080, 2005

15. Pallante P, Visone R, Ferracin M, et al: MicroRNA deregulation in human thyroid papillary carcinomas. Endocr Relat Cancer 13 497-508, 2006

16. Tetzlaff MT, Liu A, Xu X, et al: Differential expression of miRNAs in papillary thyroid carcinoma compared to multinodular goiter using formalin fixed paraffin embedded tissues. Endocr Pathol 18: 163-173, 2007.

17. Chen YT, Kitabayashi N, Zhou XK, Fahey TJ III and Scognamiglio T: MicroRNA analysis as a potential diagnostic tool for papillary thyroid carcinoma. Mod Pathol 21: 1139-1146, 2008.
18. Chou CK, Chen RF, Chou FF, et al: $\mathrm{miR}-146 \mathrm{~b}$ is highly expressed in adult papillary thyroid carcinomas with high risk features including extrathyroidal invasion and the BRAF(V600E) mutation. Thyroid 20: 489-494, 2010.

19. Cahill S, Smyth P, Finn SP, et al: Effect of ret/PTC 1 rearrangement on transcription and post-transcriptional regulation in a papillary thyroid carcinoma model. Mol Cancer 5: 70, 2006.

20. Chung KW, Kim SW and Kim SW: Gene expression profiling of papillary thyroid carcinomas in Korean patients by oligonucleotide microarrays. J Korean Surg Soc 82: 271-280, 2012.

21. Tomei S, Mazzanti C, Marchetti I, et al: c-KIT receptor expression is strictly associated with the biological behaviour of thyroid nodules. J Transl Med 10: 7, 2012.

22. Natali PG, Berlingieri MT, Nicotra MR, et al: Transformation of thyroid epithelium is associated with loss of c-kit receptor. Cancer Res 55: 1787-1791, 1995.

23. Jacques C, Baris O, Prunier-Mirebeau D, et al: Two-step differential expression analysis reveals a new set of genes involved in thyroid oncocytic tumors. J Clin Endocrinol Metab 90 2314-2320, 2005.

24. Ashman LK: The biology of stem cell factor and its receptor C-kit. Int J Biochem Cell Biol 31: 1037-1051, 1999.

25. Kitamura Y and Hirotab S: Kit as a human oncogenic tyrosine kinase. Cell Mol Life Sci 61: 2924-2931, 2004

26. Giordano S, Di Renzo MF, Narsimhan RP, Cooper CS, Rosa C and Comoglio PM: Biosynthesis of the protein encoded by the c-met proto-oncogene. Oncogene 4: 1383-1388, 1989.

27. Ruppert AM, Beau-Faller M, Belmont L, et al: A simple view on lung cancer biology: the MET pathway. Rev Mal Respir 28: 1241-1249, 2011 (In French).

28. Scartozzi M, Loretelli C, Bearzi I, et al: Allele polymorphisms of tumor integrins correlate with peritoneal carcinosis capability of gastric cancer cells in radically resected patients. Ann Oncol 22: 897-902, 2011

29. Reis PP, Waldron L, Perez-Ordonez B, et al: A gene signature in histologically normal surgical margins is predictive of oral carcinoma recurrence. BMC Cancer 11: 437, 2011.

30. Lenci RE, Rachakonda PS, Kubarenko AV, et al: Integrin genes and susceptibility to human melanoma. Mutagenesis 27: 367-373, 2012.

31. Hood JD and Cheresh DA: Role of integrins in cell invasion and migration. Nat Rev Cancer 2: 91-100, 2002.

32. Hynes RO: Integrins: versatility, modulation, and signaling in cell adhesion. Cell 69: 11-25, 1992.

33. Lu JG, Li Y, Li L and Kan X: Overexpression of osteopontin and integrin alphav in laryngeal and hypopharyngeal carcinomas associated with differentiation and metastasis. J Cancer Res Clin Oncol 137: 1613-1618, 2011.

34. Brunet A, Bonni A, Zigmond MJ, et al: Akt promotes cell survival by phosphorylating and inhibiting a Forkhead transcription factor. Cell 96: 857-868, 1999.

35. Schmid S, Bieber M, Zhang F, et al: Wnt and hedgehog gene pathway expression in serous ovarian cancer. Int J Gynecol Cancer 21: 975-980, 2011.

36. Sastre-Perona A and Santisteban P: Role of the Wnt pathway in thyroid cancer. Front Endocrinol 3: 31, 2012. 